Interactive Virtual Reality (IVR) allows trainees to not only be immersed in a simulation but take control, choosing the direction of questioning for example. It also allows the training to be easily repeated and scaled to any number of students, anytime and anywhere there is an internet connection.

Method. Following successful funding from the RCPsych General Adult Faculty we chose three common scenarios that a new started in Psychiatry would face. These included acute agitation/ rapid tranquilisation, a patient wishing to leave/section 5(2) and a patient with tachycardia following clozapine initiation. Using established guidelines and literature, in conjunction with feedback from subject matter experts and practicing clinicians, scenarios were written. We then researched the best hardware and software to make this possible, ensuring that the resources required were realistic to allow accessibility to as many trainees as possible.

Result. Creating IVR is challenging but an engaging medium. Achieving consensus on the training material is time consuming yet paramount to a good training session. Producing high quality videos is extremely resource intensive requiring large amounts of computing power and storage. However, the outcome is an engaging and practical alternative to face to face training.

Conclusion. The possibilities for IVR for are vast. For example, trainees can practice different methods of asking questions (e.g. open vs closed) and how this affects the outcome. Training could be produced centrally and then shared, allowing best practice to be disseminated. It could improve and standardise induction, especially considering the expanding workforce. It could also improve recruitment, allowing an immersive experience of Psychiatry to those who would otherwise be unable to obtain shadowing. It also has a role in patient safety - demonstrating common scenarios that the trainee may face allowing them to practice in a safe environment.

Patterns of antipsychotic prescribing in first episode psychosis - differences between acute and early intervention services

James Fallon $^{1 \star}$, Sophie McBrien ${ }^{2}$ and Keegan Curlewis ${ }^{2}$

${ }^{1}$ Sussex Partnership NHS Trust and ${ }^{2}$ Brighton and Sussex Medical School

${ }^{\star}$ Corresponding author.

doi: 10.1192/bjo.2021.116

Aims. This study aimed to evaluate the patterns of antipsychotic prescribing in patients with first episode psychosis (FEP) at the time of their initial treatment and over the first year with the Early Intervention Service (EIS). It was hypothesised that different care teams would have a preference for certain antipsychotic medications and that initial medication choice would be continued through the first year.

Background. Research indicates that with the exception of clozapine, all antipsychotics are equally as effective. However, anecdotally it has been observed that inpatient and crisis teams and EIS have differing initial medication choices.

Method. An analysis of the North West Sussex EIS caseload $(n=67)$ was conducted. The first antipsychotic prescribed and initiating team was recorded. Prescribed medication for those that had completed 12 months $(\mathrm{n}=43)$ with EIS after initial prescription was recorded. An analysis was performed of prescribing choice by initial care team (acute vs EIS vs other community services) with the frequency with which medication was changed during treatment.

Result. $97 \%(n=65)$ of patients were started on an antipsychotic. Initial medication choice was olanzapine $(44.8 \%, \mathrm{n}=30)$, aripiprazole $(22.4 \%, \mathrm{n}=15)$, risperidone $(20.9 \%, \mathrm{n}=14)$, quetiapine $(6 \%, \mathrm{n}=4)$ and zuclopenthixol were least common $(1.5 \%, \mathrm{n}=$ $2)$. At the 12 month point $51.2 \%(n=22$ of 43$)$ had switched and $16.3 \%$ ( $n=7$ of 43 ) had discontinued.

The most common medication started by acute services was olanzapine $(56.0 \%, \mathrm{n}=28$ of 50$)$, though of those who completed 12 months this had been switched in $53 \%$ of cases $(n=9$ of 17$)$. EIS most commonly initiated aripiprazole or risperidone $(37.5 \%$ each $n=4)$. At 6 and 12 month follow-up by EIS, the most commonly prescribed antipsychotic was aripiprazole (24 patients $40.7 \%$, and 14 patients $32.6 \%$ respectively).

Conclusion. There was a clear preference for olanzapine as initial treatment of First Episode of Psychosis in the region. On breakdown it was apparent that there was a split in prescribing choices between more sedating medication in acute services and less sedating medication in EIS. Given that most patients changed to less sedating and less metabolic active medications over their first year it is not clear why alternative options are not used at the start of treatment. Future research will focus on clinician's rationale for initial prescribing choice. This will look for any underlying bias toward specific medications.

\section{Eating disorders and psychosis: a case report and review of the literature}

\section{Defne Flora Goy*, Erdem Efe, Özge Şahmelikoğlu} and Ümit Haluk Yeşilkaya

Bakirkoy Research \& Training Hospital for Psychiatry, Neurology and Neurosurgery

${ }^{\star}$ Corresponding author.

doi: 10.1192/bjo.2021.117

Aims. Despite evidence from case series, the comorbidity of eating disorders (ED) with psychosis is a challenging field to which little attention has been paid. There is no consistent sequence in the co-occurrence of the two conditions-eating disorders sometimes precede, and sometimes follow the onset of psychosis. In this case report, we present a 25 -year-old female patient suffering from recurrent episodes of binge eating and inappropriate compensatory purging behaviours with psychotic components to discuss the co-occurrence of these conditions in the light of the literature.

Method. Our patient suffered from sleep disturbances, homicidal thoughts, self-induced vomiting worsened in one year. Psychiatric examination revealed psychotic symptoms such as blunted affect, persecutory delusions, and delusions of appeal and justification. In our inpatient psychiatry clinic, she was treated with olanzapine 20 milligrams(mg) and quetiapine $500 \mathrm{mg}$ per day.

Result. Psychotic episodes occur in $10-15 \%$ of eating disorder patients. The prevalence of primary psychotic diseases like schizophrenia and schizoaffective disorders in eating disorder patients appears to be comparable to that in the general population. An ED can be the early sign of an impending psychosis, or psychotic symptoms can signal the beginning of an ED. The advent of the psychosis, and sometimes the treatment of the psychosis can cure the eating disorder, but it can sometimes aggravate it. The case presented illustrates the difficulties in managing a patient with a comorbid eating disorder and psychosis. To ensure a rigorous assessment of both psychotic and 